\title{
On the Shape of the Mesopores in Zeolite Y: A Three-Dimensional Transmission Electron Microscopy Study Combined with Texture Analysis
}

\author{
A. H. Janssen, ${ }^{\dagger}$ A. J. Koster, \\ Department of Inorganic Chemistry and Catalysis, Debye Institute, Utrecht University, PO Box 80083, \\ 3508 TB Utrecht, The Netherlands, and Department of Molecular Cell Biology, Utrecht University, \\ Padualaan 8, 3584 CH Utrecht, The Netherlands
}

Received: April 19, 2002

\begin{abstract}
The shape of the mesopores in a series of $\mathrm{Y}$ zeolites modified with different postsynthesis treatments was investigated. A combination of 3D-TEM (3D-TEM = three-dimensional transmission electron microscopy) with texture analysis (nitrogen physisorption and mercury porosimetry) enabled us to discriminate between cylindrical mesopores and mesoporous cavities inside the zeolite that are connected to the external surface via micropores. A large part of the mesopores in steamed and acid leached zeolite Y proved to be cavities, although the cylindrical mesopores in these samples account for the majority of the mesopore volume. Only after special hydrothermal treatments a zeolite $\mathrm{Y}$ with almost exclusively interconnected cylindrical mesopores is obtained.
\end{abstract}

\section{Introduction}

Zeolites are microporous crystalline aluminosilicates, which makes them very suitable for separation processes or shape selective catalysis. ${ }^{1}$ For a number of industrial catalytic applications - catalytic cracking, hydrocracking, aromatic alkylation, and alkane hydroisomerization-it has been demonstrated that the presence of mesopores in microporous zeolite crystals is essential to alleviate diffusion limitation. ${ }^{2-4} \mathrm{~A}$ widely applied method to generate mesopores involves steaming and acidleaching treatments of zeolite crystals. These treatments generate mesopores by extraction of aluminum from the zeolite lattice, thus causing partial collapse of the framework. ${ }^{5,6}$ However, the mesopores formed during steaming are predominantly cavities inside the zeolite crystals, rather than cylindrical pores connecting the external surface with the interior of the crystal. ${ }^{7}$ This implies that diffusion of reactants through the crystals is increased by the steaming and acid leaching procedure, but not as much as it would have been in the case of cylindrical mesopores connected to the external surface (see Figure 1).

In U.S. patent 5601798 (PQ Corp) a method is described to prepare zeolite $\mathrm{Y}$ with very high mesopore volumes $(0.3-0.6$ $\mathrm{mL} / \mathrm{g}$ ) via a hydrothermal treatment. We have characterized a thus prepared high-meso VUSY (very ultra stable Y) zeolite with nitrogen physisorption, ${ }^{8-10}$ mercury porosimetry, ${ }^{11,12}$ and three-dimensional transmission electron microscopy (3D-TEM) ${ }^{13}$ and compared the results with steamed and acid leached zeolite $\mathrm{Y}$ in order to discriminate between cylindrical mesopores and mesoporous cavities inside the zeolite crystals.

Most often mercury intrusion is used to measure the macroand larger mesopores between the individual crystals of a material. Here we use mercury intrusion to discriminate between cylindrical mesopores in the zeolite crystals connected to the external surface and mesoporous cavities inside the crystals connected to the external surface by micropores. At the pressures

Department of Inorganic Chemistry and Catalysis.

Department of Molecular Cell Biology. applied, mercury cannot intrude in pores smaller than $3.7 \mathrm{~nm}$ diameter. Since the cavities inside the zeolite are connected to the external surface only by micropores of $0.7 \mathrm{~nm}$ diameter, mercury will not intrude in these cavities. However, the cylindrical mesopores with diameters larger than $3.7 \mathrm{~nm}$ that reach the external surface of the crystals will be filled with mercury. Comparing the mercury intrusion results with nitrogen physisorption enables us to discriminate between the volumes of the cylindrical and cavity-type mesopores.

In this paper we compare the shape of the mesopores in a series of $\mathrm{Y}$ zeolites modified with different postsynthesis treatments. A combination of 3D-TEM with texture analysis (nitrogen physisorption and mercury porosimetry) enabled us to discriminate between cylindrical mesopores and mesoporous cavities inside the zeolite that are connected to the external surface via micropores.

\section{Experimental Section}

Samples CBV100 (NaY), CBV400 (Ultra Stable Y, steamed once) and CBV780 (eXtra Very Ultra Stable Y, steamed twice and acid leached) and a sample of High-Meso Very Ultra Stable Y were obtained from Zeolyst and PQ Corp. Nitrogen adsorption and desorption measurements were performed at liquid nitrogen temperature on a Micromeritics ASAP 2010. Mercury intrusion measurements were performed on a Pascal 404 (Thermo Finnigan) to a final pressure of $400 \mathrm{MPa}$. For electron microscopy a droplet of a colloidal gold suspension (Sigma, 5 $\mathrm{nm}$ gold) was dried on a carbon coated copper grid, thus providing markers for the alignment of the data set. ${ }^{13}$ Next, a droplet of a suspension of the sample in ethanol was dried on this grid. From a representative crystal a tilt series of ca. 131 images $(1024 \times 1024$ pixels $)$ was taken from about $+65^{\circ}$ to $-65^{\circ}$ with $1^{\circ}$ intervals at a magnification of $14.5 \mathrm{k}(1$ pixel $=$ $0.97 \mathrm{~nm})$ or $19 \mathrm{k}(1$ pixel $=0.74 \mathrm{~nm})$ on a Tecnai 20 microscope $(200 \mathrm{kV})$, using software for automated electron tomography. ${ }^{14,15}$ From the tilt series a 3D-reconstruction of the crystal is calculated as a stack of thin $(1-2 \mathrm{~nm})$ slices. 

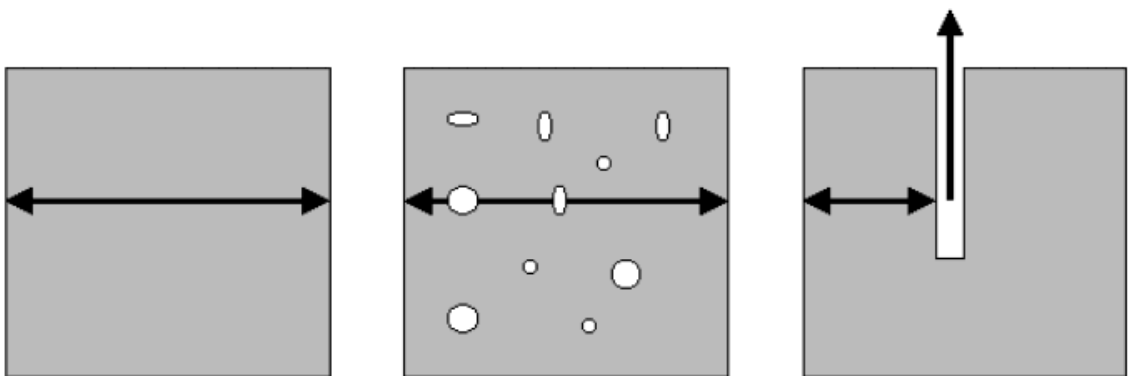

Figure 1. Diffusion length in the micropores of a zeolite crystal. Left: without mesopores. Middle: small decrease by mesoporous cavities. Right: large decrease by cylindrical mesopores connected to the external surface.

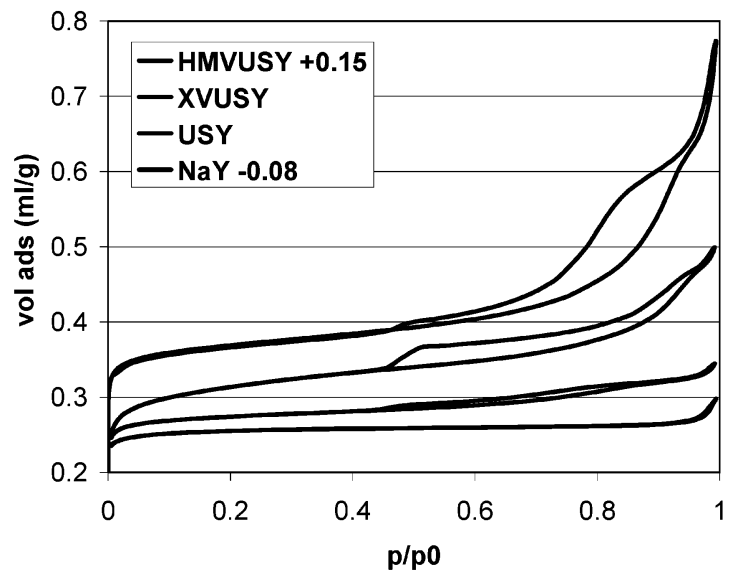

Figure 2. Nitrogen adsorption and desorption isotherms (relative $\mathrm{N}_{2}$ pressure $\mathrm{p} / \mathrm{p} 0$ against adsorbed volume) for several $\mathrm{Y}$ zeolites (from top to bottom: HMVUSY, XVUSY, USY and NaY). For clarity the isotherm of $\mathrm{NaY}$ has been shifted $0.08 \mathrm{~mL} / \mathrm{g}$ downward and the isotherm of the HMVUSY has been shifted upward by $0.15 \mathrm{~mL} / \mathrm{g}$.

\section{Results and Discussion}

In Figure 2 the nitrogen adsorption and desorption isotherms are given for the different $\mathrm{Y}$ zeolites. From these data it is clear that the mesopore system of the high-meso VUSY (HMVUSY) differs very much from the mesopore systems in the steamed and acid leached zeolite Y's (USY and XVUSY). Not only the mesopore volume is much larger (see also Table 1) but also the shape of the hysteresis loop is very different. For USY and XVUSY, the hysteresis loop is rather flat, indicating inkbottle type pores, whereas the upward curvature of the hysteresis loop of HMVUSY is indicative of cylindrical type of pores. In Figure $3 \mathrm{~b}$ the pore size distribution is given as calculated from the desorption branch with the BJH-method. From this figure it is

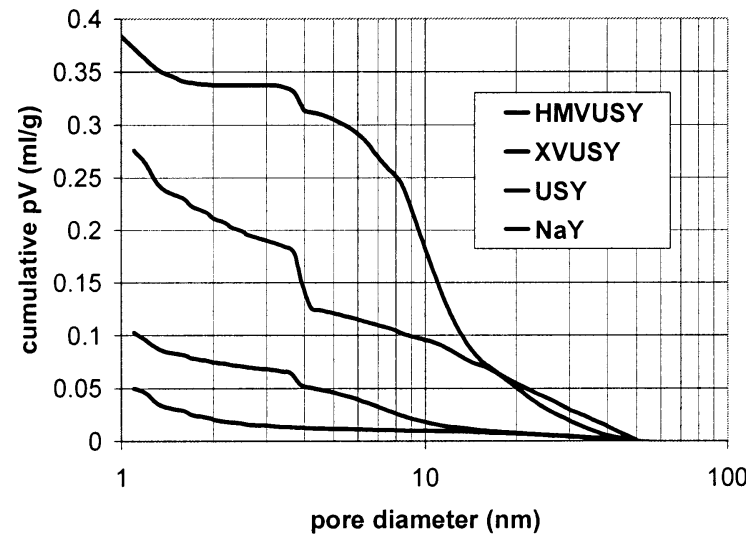

TABLE 1: Physical Properties of NaY (CBV100), USY (CBV400), HMVUSY and XVUSY (CBV780)

\begin{tabular}{lrrcrcccr}
\hline & $\begin{array}{c}\text { Si/Al } \\
\text { bulk } \\
\text { (at/at) }\end{array}$ & $\begin{array}{c}\text { Si/Al } \\
\text { XPS } \\
(\text { at/at })\end{array}$ & $\begin{array}{c}a_{0} \\
(\mathrm{~nm})\end{array}$ & $\% \mathrm{Y}^{a}$ & $\begin{array}{c}V_{\text {micro }}{ }^{b} \\
(\mathrm{~mL} / \mathrm{g})\end{array}$ & $\begin{array}{c}V_{\text {micro }} \\
\text { calcd }^{c} \\
(\mathrm{~mL} / \mathrm{g})\end{array}$ & $\begin{array}{c}V_{\text {meso }}{ }^{d} \\
(\mathrm{~mL} / \mathrm{g})\end{array}$ & $\begin{array}{c}S_{\mathrm{T}}{ }^{e} \\
\left(\mathrm{~m}^{2} / \mathrm{g}\right)\end{array}$ \\
\hline $\mathrm{NaY}$ & 2.6 & 2.8 & 2.469 & 100 & 0.34 & 0.34 & 0.05 & 8 \\
USY & 2.6 & 1.1 & 2.450 & 87 & 0.26 & 0.32 & 0.11 & 63 \\
XVUSY & 39.3 & 71.3 & 2.423 & 72 & 0.28 & 0.27 & 0.25 & 120 \\
HMVUSY & 5.0 & 1.4 & 2.427 & 71 & 0.15 & 0.26 & 0.47 & 146
\end{tabular}

${ }^{a}$ Relative crystallinity. ${ }^{b}$ Micropore volume from $t$-plot. ${ }^{c}$ Expected micropore volume if all micropores are empty; calculated by correcting $V_{\text {micro }}$ of NaY for crystallinity, $a_{0}$ and sodium weight of USY and XVUSY. ${ }^{d} V_{\text {total }}-V_{\text {micro }}{ }^{e}$ Sum of external and mesopore surface area calculated from $t$-plot.

clear that the size of the mesopores increases in the order: USY $(2-20 \mathrm{~nm})$, HMVUSY $(4-25 \mathrm{~nm})$, XVUSY $(4-40 \mathrm{~nm})$. The peak at a diameter of $3.5 \mathrm{~nm}$ (indicated with “*”) is an artifact of the BJH-method. At a relative pressure of ca. 0.45 there is a sudden desorption of nitrogen, which is visible as the closing of the hysteresis loop at this pressure. All inkbottle type mesopores with pore necks smaller than $4 \mathrm{~nm}$ will release their nitrogen at a relative pressure of ca. 0.45 . Therefore, the amount of nitrogen desorbing at a relative pressure of ca. 0.45 is real, but it cannot be ascribed to pores of $3.5 \mathrm{~nm}$ diameter alone (as calculated with the BJH-method).

Also the micropore volume of the high-meso VUSY is much lower than the other three samples: $0.15 \mathrm{~mL} / \mathrm{g}$ vs $0.34 \mathrm{~mL} / \mathrm{g}$ for $\mathrm{NaY}$. This is mainly caused by a blocking of the micropores with material generated during the hydrothermal treatment, just as (to a lesser extent) is the case with USY. When the measured micropore volume is compared with the calculated micropore volume that is expected on the basis of the crystallinity, sodium content and unit cell size of the materials (see Table 1: $V_{\text {micro }}$ and $V_{\text {micro }}$ calcd), the pore blockage is evident.

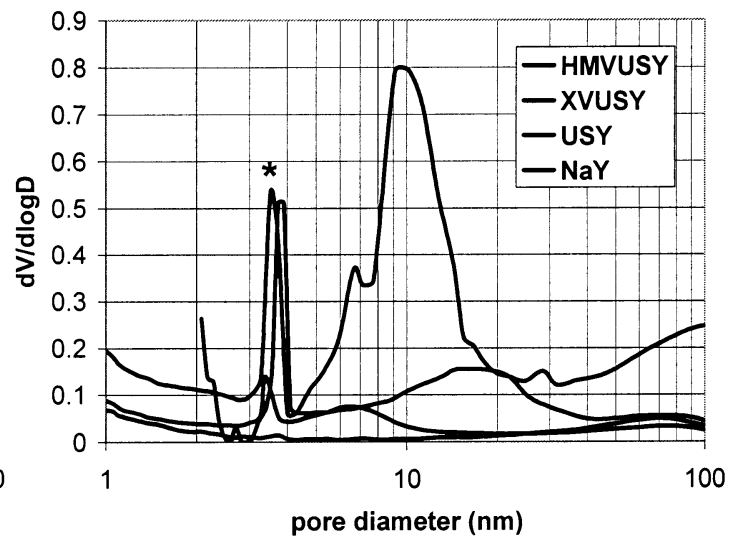

Figure 3. Nitrogen physisorption on HMVUSY, XVUSY, USY and NaY (from top to bottom). a: Cumulative desorption volume vs pore diameter scaled to 0 at a diameter of $50 \mathrm{~nm}$. b: Pore size distributions (pore diameter $D$ against $\mathrm{d} V / \mathrm{d} \log D$ (a measure for the number of pores of certain diameter)) calculated from the nitrogen desorption isotherms (BJH method). The peak indicated by "*" is an artifact (see text). 

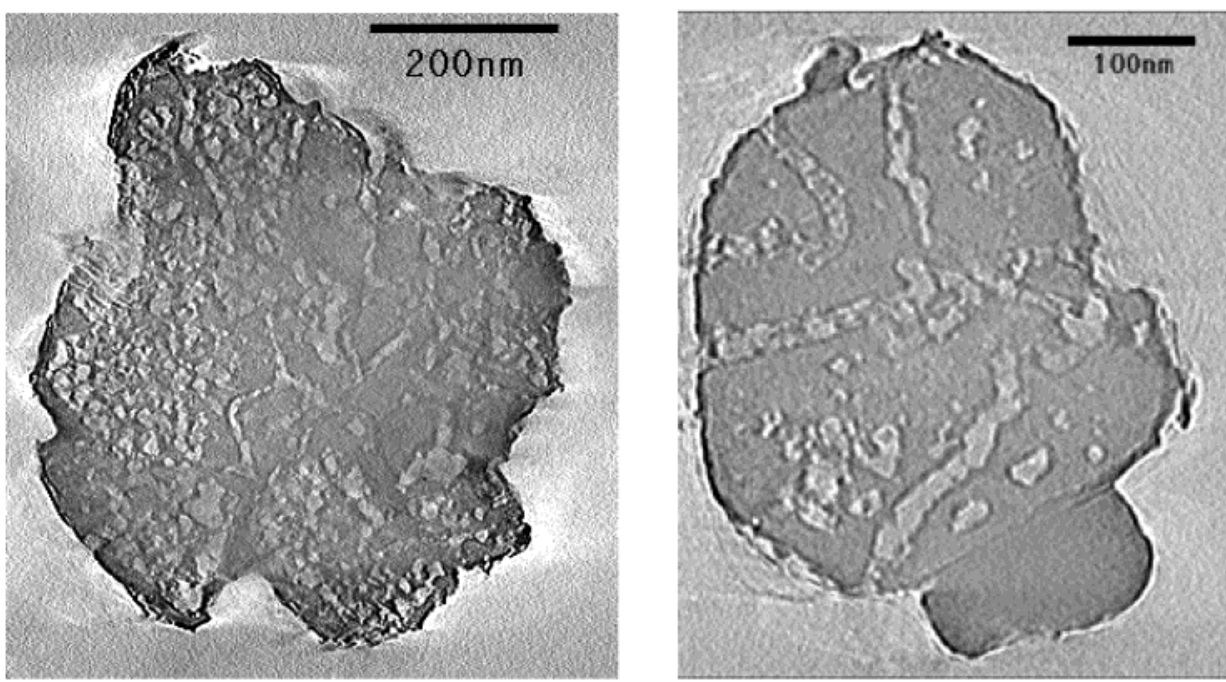

Figure 4. 3D-TEM slices of two HMVUSY crystals, showing the interconnected cylindrical mesopore system.
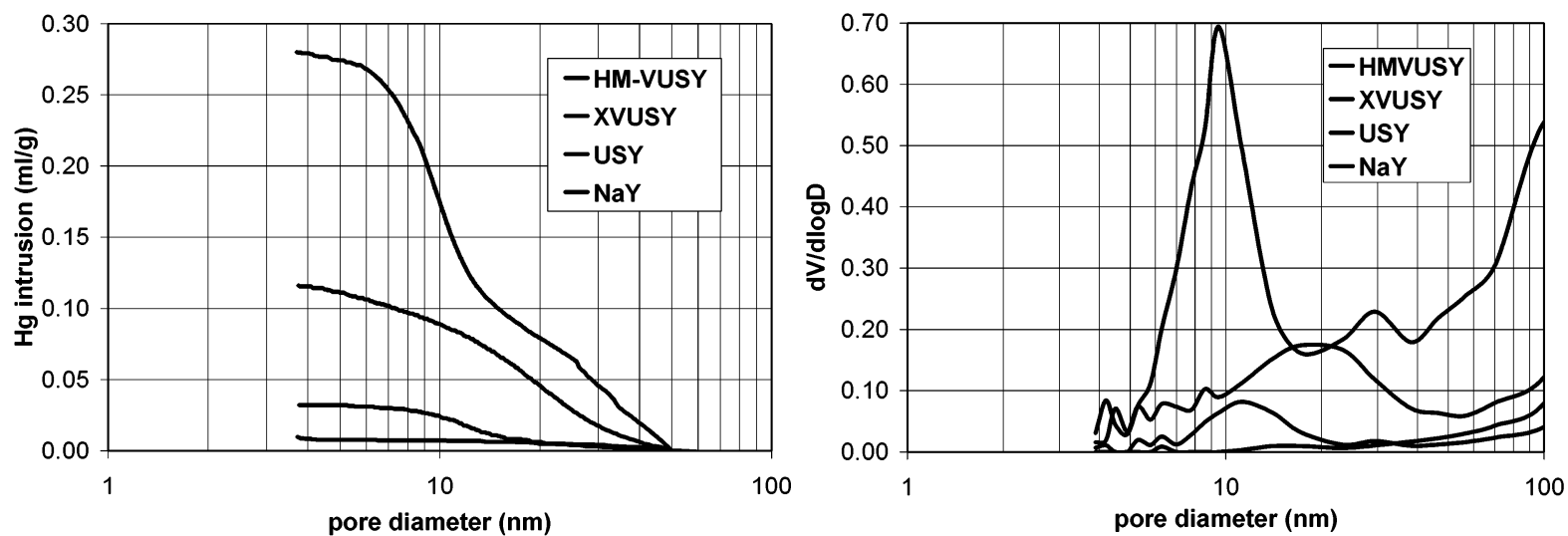

Figure 5. Hg-intrusion for HMVUSY, XVUSY, USY and NaY (from top to bottom). a: Cumulative Hg-intrusion vs pore diameter (calculated with the Washburn equation), scaled with intrusion $=0$ at a diameter of $50 \mathrm{~nm}$. b: Pore size distribution [pore diameter $D$ against $\mathrm{d} V / \mathrm{d} \log \mathrm{D}(\mathrm{a}$ measure for the number of pores of certain diameter)].

In Figure 4 two 3D-TEM slices through the reconstructions of two HMVUSY crystals are given. The very high porosity due to the mesopore system is clearly visible. By studying the subsequent slices through the $3 \mathrm{D}$ reconstruction it is clear that the mesopore system consists of interconnected cylindrical mesopores. Despite the very porous nature of this zeolite, the peaks in the XRD pattern (not shown) are still very sharp. This indicates that the crystal is still a single crystal, although there are many pores in it, rather than many crystallites bound together.

In Figure 5 the $\mathrm{Hg}$-intrusion data of the four zeolite $\mathrm{Y}$ samples is given. In Figure $5 \mathrm{a}$ the intrusion is scaled at $0 \mathrm{~mL} / \mathrm{g}$ intrusion at a pore diameter of $50 \mathrm{~nm}$ to allow a better comparison of the $\mathrm{Hg}$-intrusion in the mesopores, thus discarding the intrusion in the macropores. When comparing the cumulative mercury intrusion (Figure 5a) with the cumulative nitrogen desorption (Figure 3a) it is clear that there are many similarities. For $\mathrm{NaY}$, in the pressure ranges considered, there is no mercury intrusion and also no nitrogen desorption, because there are no mesopores. For XVUSY the cumulative pore volumes from mercury intrusion and nitrogen desorption are almost the same up to 4 $\mathrm{nm}$ (the lower limit for mercury intrusion). For HMVUSY, the total mercury intrusion volume is lower than the desorbed volume of nitrogen, but the shape of the curves is similar. The fact that the shape of the mercury intrusion curves and the nitrogen desorption curves coincides for NaY, XVUSY and HMVUSY, is reflected in their pore size distributions (compare

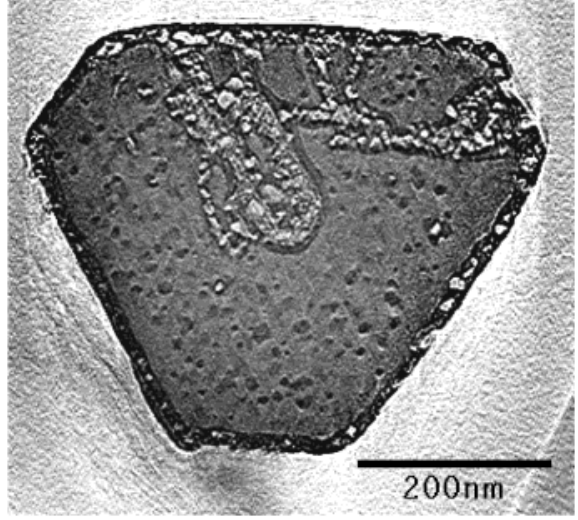

Figure 6. 3D-TEM slice $(1.7 \mathrm{~nm})$ through a USY crystal.

Figure $3 b$ and $5 b$ ). For NaY, XVUSY, and HMVUSY the pore size distribution calculated from mercury intrusion is equal to the pore size distribution calculated from the nitrogen desorption data. For USY the mercury intrusion volume is almost equal to the desorbed nitrogen volume, but the mercury intrusion takes place at somewhat larger pore diameters according to Figures $3 \mathrm{~b}$ and $5 \mathrm{~b}$. One possible explanation for the different pore diameters could lie in the fact that we have used a fixed value for the contact angle (between the mercury and the zeolite) in the Washburn equation that was used to calculate the pore diameters. If the contact angle of the mercury on the USY differs 
TABLE 2: Mesopore Volumes from Cumulative Nitrogen Physisorption and Mercury Intrusion

\begin{tabular}{lcccccc}
\hline sample & $\begin{array}{c}\text { mesopore } \\
\text { diameters } \\
(\mathrm{nm})\end{array}$ & $\begin{array}{c}V_{\text {meso }} \\
(\mathrm{mL} / \mathrm{g})^{a}\end{array}$ & $\begin{array}{c}V_{\text {meso }} \\
\mathrm{N}_{2} \\
(\mathrm{~mL} / \mathrm{g})^{b}\end{array}$ & $\begin{array}{c}V_{p / p_{0}=0.45} \\
\mathrm{~N}_{2} \\
(\mathrm{~mL} / \mathrm{g})^{c}\end{array}$ & $\begin{array}{c}V_{\text {tot }}- \\
V_{\text {micro }} \\
(\mathrm{mL} / \mathrm{g})^{d}\end{array}$ & $\begin{array}{c}V \\
\text { cavities } \\
(\%)^{e}\end{array}$ \\
\hline $\mathrm{NaY}$ & $\mathrm{X}$ & 0 & 0 & 0 & 0.05 & \\
USY & $4-20$ & 0.03 & 0.04 & 0.01 & 0.11 & 20 \\
XVUSY & $4-40$ & 0.12 & 0.12 & 0.05 & 0.25 & 29 \\
HMVUSY & $4-25$ & 0.22 & 0.26 & 0.02 & 0.47 & 7
\end{tabular}

${ }^{a}$ Mercury intrusion volume in pores with diameters specified in the second column. ${ }^{b} \mathrm{BJH}$ cumulative nitrogen desorption from pores with diameters specified in the second column. ${ }^{c}$ Nitrogen volume desorbing at the step around $\mathrm{p} / \mathrm{p}_{0}=0.45$ in the cumulative nitrogen desorption. ${ }^{d} V_{\text {macro }}+V_{\text {meso. }} \cdot{ }^{e} V_{p / p_{0}=0.45} /\left(V_{\text {meso }} \mathrm{N}_{2}+V_{p / p_{0}=0.45}\right)$.

from the contact angle on the XVUSY and HMVUSY, which have much higher $\mathrm{Si} / \mathrm{Al}$ ratios, this can give an explanation why the shape of the mercury intrusion curves coincide with the cumulative nitrogen desorption curves for XVUSY and HMVUSY but not for USY. However, since we have not measured the contact angles, we can only speculate on this. One other point is the difference in mercury intrusion volume for HMVUSY compared to the nitrogen desorption volume $(0.22$ and $0.26 \mathrm{~mL} / \mathrm{g}$, respectively). We do not know where this difference comes from. However, taking into account that the two techniques are rather different in nature, there is still a reasonable agreement between the volumes.

By comparing the results obtained with mercury intrusion and nitrogen physisorption, one can now discriminate between the cylindrical part of the mesopore system and the mesoporous cavities in the crystal. In Table 2 the measured nitrogen and mercury volumes are given. It has been shown before that for USY and XVUSY there is a good agreement between the pore sizes calculated from the nitrogen desorption measurements and the pore sizes measured from the 3D-TEM reconstructions. ${ }^{7}$ The current 3D-TEM reconstructions of HMVUSY crystals (Figure 4) also show pore dimensions in good agreement with the pore sizes calculated from the nitrogen desorption data. Therefore, the range of mesopore diameters is taken from the pore size distribution calculated from the nitrogen desorption branch. When the volume of nitrogen desorbing in the specified range is compared with the volume of mercury intruded in pores with the same range, there is a reasonable to good agreement (compare $V_{\text {meso }} \mathrm{Hg}$ with $V_{\text {meso }} \mathrm{N}_{2}$ in Table 2). This indicates that this is the volume of cylindrical mesopores connected to the external surface of the zeolite crystals. The many cavities observed with 3D-TEM behave like inkbottle pores because they are connected to the external surface via the micropores. Thus, the amount of nitrogen desorbing from inkbottle type of pores gives us a good indication of the total volume of the mesoporous cavities. This amount of nitrogen is released at once and can be seen as a step in the nitrogen desorption isotherm (around a relative pressure of 0.45 ) and as a step in the cumulative desorption plot (pore diameters of 3-4 nm). With the volume of the mesoporous cavities (from the step at a relative pressure of 0.45 ) and the total mesopore volume known, the relative fraction of the mesopore volume present as cavities can be calculated. The relative fraction of the cavities with respect to the total mesopore volume is $0.20,0.29$, and 0.07 for USY, XVUSY, and HMVUSY respectively (see Table 2). From these values it can be concluded that although there are many cavities in the USY and XVUSY samples (as can be seen with 3DTEM), the majority of the total mesopore volume is still located in cylindrical mesopores. After hydrothermal treatment, the number of mesoporous cavities is much lower than after steaming. During steaming aluminum ions are extracted from the framework, leaving lattice vacancies. Part of these vacancies are healed by mobile silicon species, whereas others act as a source of silicon species, resulting in the formation of mesopores. ${ }^{16}$ In places with high concentrations of vacancies or cavities, the cavities can coalesce, resulting in the formation of cylindrical mesopores. ${ }^{17}$ We can only speculate what happens during the hydrothermal treatment that leads to the HMVUSY. Most likely, the sample subjected to the hydrothermal treatment has been strongly dealuminated by steaming and acid leaching. The very high aluminum content on the external surface of the HMVUSY sample can result from the use of USY as a starting material or from the use of an aqueous sodium aluminate solution. When such a starting material, with both cylindrical mesopores and a large number of cavities, is subjected to a hydrothermal treatment part of the zeolite is dissolved. Most likely this takes place from areas where the zeolite framework already contains defects: the edges of the mesopores. This results in an increase in the diameters of the mesopores as well as the coalescence of several cavities to cylindrical mesopores.

From the above we conclude that the mesopore system can be tuned to some extent by the different hydrothermal and acid treatments. However, during these treatments also the zeolite itself is changed by extraction of aluminum and (unwanted) deposition of amorphous material inside the micro- and mesopores. This is an incentive for the generation and tuning of mesopores during the synthesis of the crystallites. One of the promising ways to direct the size and shape of the mesopore system has recently been shown by Jacobsen et al. ${ }^{18,19}$ By using a carbon source as a mesopore templating agent during zeolite synthesis and subsequent calcination, they obtained a zeolite with a mesopore system. Finally, it is advocated that alternative physisorption theories, such as $\mathrm{NLDFT}^{9}$ will be considered to refine the description of mesopores in zeolite crystals.

\section{Conclusions}

By combining the results obtained with 3D-TEM, nitrogen physisorption and mercury intrusion of several zeolite $\mathrm{Y}$ samples, a discrimination can be made between cylindrical mesopores connected to the external surface of the crystals and mesoporous cavities inside the crystals that are connected to the external surface only by micropores. After steaming (and acid leaching) there are many mesoporous cavities, although on a volume basis, the majority of the mesopore volume is in cylindrical mesopores. After a special hydrothermal treatment zeolite Y crystals with very high mesopore volumes are obtained. The mesopore system thus obtained consists predominantly of interconnected cylindrical mesopores, although there are still some cavities left. Such a system of interconnected cylindrical mesopores is expected to enhance the diffusion much more than mesoporous cavities inside the crystals.

Hydrothermal and acid leaching treatments are successful to obtain highly mesoporous zeolite $\mathrm{Y}$ crystals. While mild treatments result in many cavities inside the crystals, more severe treatments cause a loss of crystallinity and a decrease of micropore volume. Very often these treatments also result in the (unwanted) deposition of material inside the micro- and mesopores. This is an incentive for the generation and tuning of mesopores during the synthesis of the crystallites.

Acknowledgment. Supported by NWO/CW under Grant 98037. The research of A.J.K. has been made possible by a fellowship of the Royal Netherlands Academy of Arts and Sciences (KNAW). The authors thank M. Mittelmeijer of the 
University of Amsterdam for mercury porosimetry measurements, J.A.R. van Veen and E.J. Creyghton for physical data and useful discussions and Zeolyst and PQ Corp. for the samples.

Supporting Information Available: Moving images of the consecutive slices through the three-dimensional reconstruction of one of the crystals of Figure 4. This material is available free of charge via the Internet at http://pubs.acs.org.

\section{References and Notes}

(1) Marcilly, C. R. Top. Catal. 2000, 13, 357-366.

(2) Tromp, M.; van Bokhoven, J. A.; Garriga Oostenbrink, M. T.; Bitter,

J. H.; de Jong, K. P.; Koningsberger, D. C. J. Catal. 2000, 190, 209-214.

(3) van Donk, S.; Broersma, A.; Gijzeman, O. L. J.; van Bokhoven, J.

A.; Bitter, J. H.; de Jong, K. P. J. Catal. 2001, 204, 272-280.

(4) Meima, G. R. CATTECH 1998, 2, 5-12.

(5) Scherzer, J. ACS Symp. Ser. 1984, 248, 157-200.

(6) Beyerlein, R. A.; Choi-Feng, C.; Hall, J. B.; Huggins, B. J.; Ray, G. J. Top. Catal. 1997, 4, 27-42.

(7) Janssen, A. H.; Koster, A. J.; de Jong, K. P. Angew. Chem., Int. Ed. 2001, 40, 1102-1104.
(8) Kruk, M.; Antochshuk, V.; Matos, J. R.; Mercuri, L. P.; Jaroniec, M. J. Am. Chem. Soc. 2002, 124, 768-769.

(9) Ravikovitch, P. I.; Wei, D.; Chueh, W. T.; Haller, G. L.; Neimark, A. V. J. Phys. Chem. B 1997, 101, 3671-3679.

(10) Hudec, P.; Jorik, V.; Smieskova, A.; Zidek, Z. React. Kinet. Catal. Lett. 1997, 15-19.

(11) Leon y Leon, C. A. Adv. Colloid Interface Sci. 1998, 76-77, 341372.

(12) Rouquerol, J.; Avnir, D.; Fairbridge, C. W.; Everett, D. H.; Haynes, J. H.; Pernicone, N.; Ramsay, J. D. F.; Sing, K. S. W.; Unger, K. K. Pure Appl. Chem. 1994, 66, 1739-1758.

(13) Koster, A. J.; Ziese, U.; Verkleij, A. J.; Janssen, A. H.; de Jong, K. P. J. Phys. Chem. B 2000, 104, 9368-9370.

(14) Koster, A. J.; Grimm, R.; Typke, D.; Hegerl, R.; Stochek, A.; Walz, J.; Baumeister, W. J. Struct. Biol. 1997, 120, 276-308.

(15) Ziese, U.; Janssen, A. H.; Murk, J.-L.; Geerts, W. J. C.; van der Krift, T.; Verkleij, A. J.; Koster, A. J. J. Microscopy 2002, 205, 187-200. (16) Marcilly, C. Pet. Tech. 1986, 382, 12-18.

(17) Choi-Feng, C.; Hall, J. B.; Huggins, B. J.; Beyerlein, R. A. J. Catal. 1993, 140, 395-405.

(18) Jacobsen, C. J. H.; Madsen, C.; Houzvicka, J.; Schmidt, I.; Carlsson, A. J. Am. Chem. Soc. 2000, 122, 7116-7117.

(19) Schmidt, I.; Boisen, A.; Gustavsson, E.; Stahl, K.; Pehrson, S.; Dahl, S.; Carlsson, A.; Jacobsen, C. J. H. Chem. Mater. 2001, 13, 4416-4418. 